$\xi=-1$

\title{
Testing and Analysis of the HRV Signals from Wearable Smart HRV Sensors
}

\author{
Zigurds Markovics $^{1}$, Juris Lauznis ${ }^{1}$, Matiss Erins ${ }^{1}$, Olesja Minejeva ${ }^{1}$, Raivis Kivlenieks ${ }^{1}$ \\ ${ }^{1}$ Faculty of Computer Science and Information Technology, Riga Technical University, Latvia
}

\begin{abstract}
The objective of the test procedure is to obtain bio signals from Photoplethysmograph and Electrocardiograph sensors on selected consumer devices and to statistically validate the data for use with a drowsiness estimation method.

The method selected for validation uses LF/HF ratio calculated by a set of R-R interval data to estimate drowsiness state of a human. The value LF to HF ratio calculates balance between sympathetic and parasympathetic activity that can be measured from HRV (Heart rate variability) signals. The statistical data collected are processed by using Fast Fourier Transform and HRV frequency domain analysis on a set of test participants.

There is a correlation between medical ECG equipment control output and Matlab tool's HRVAS (Burg) output of data processed from ECG based wearable smart sensor when the LF/HF ratio is calculated in all observed volunteer data. The results for Photoplethysmograph sensors of this test correlate with other tested tools but level of the values is lower, and data from optical biosensor devices which are designed to measure HRV time-domain properties as pulse did not confirm with ECG equipment results for frequency-domain analysis required for use with selected drowsiness estimation method. The result affecting factors are sensor placement, motion artefacts and discrete vendor-specific signal pre-processing of wearable device output data.

The following results confirm the use of consumer grade biosensor that produces discretely pre-processed R-R interval data for the frequency based HRV method and application validation against directly processed ECG data from certified medical equipment.
\end{abstract}

Keywords: Heart Rate Variability, Wearable Sensor Biosignals, Photoplethysmography, Sensor Validation.

\section{Introduction}

The cardiovascular system is mostly controlled by autonomic regulation through the activity of sympatic and parasympathetic pathways of the autonomic nervous system. HRV analysis attempts to assess cardiac autonomic regulation through quantification of sinus rhythm variability. HRV, heart rate variability, is the degree of fluctuation in the length of the intervals between heart beats. (Malik \& Camm, 1995, pp. 393-406)

HRV is mirroring the regularity of hearth beats: higher regularity lowers HRV (and vice versa). Regularity of heartbeats is derived from a quantity of numbers; equal to the times elapsed between successive heartbeats. They are named R-R intervals and are measured in milliseconds. R-R intervals are obtained from ECG or plethysmography (Medicore, 2005).

Since HRV aims at quantifying autonomic regulations, it can be used as marker of sympathetic or parasympathetic predominance, and therefore become relevant in many applications. In athletes for example (Aubert, Seps \& Beckers, 2003) heavy training is responsible for shifting the cardiac autonomic balance toward a predominance of the sympathetic over the parasympathetic drive and HRV analysis attempts to quantify this shift.

Other applications span from stress monitoring during public speaking (Kusserow, Amft \& Tröster, 2008) or daily life (Vrijkotte, van Doornen \& de Geus, 2000), assessment of pathological conditions (Nolan et al., 1998) and even emotional regulation during financial decision-making (trading) (Fenton-O'Creevy et al., 2012).
Fluctuations in HR are often thought to be periodic and occurring on many time scales. Quantifying these fluctuations within the RR time series can be done by calculating the power spectrum density (PSD). The PSD presents spectral power density of a time series as a function of frequency. Typical HRV measures taken from frequency-domain analysis are powers within frequency bands and ratios of powers. The amount of power contained within a frequency band is obtained by integrating the PSD within the band frequency limits. Measures of spectral power are reported as absolute (aVL, aVF, aHF), percentage of the sum of aLF and aHF (pLF, pHF), or normalized to total power (nLF, nHF). In addition to the power measures, the ratio of LF to $\mathrm{HF}(\mathrm{LF} / \mathrm{HF})$ provides a so called sympatho-vagal balance. Finally, less commonly mentioned measures are the peak frequencies within the VLF, LF, and $\mathrm{HF}$ bands. In general, frequency-domain HRV analysis is concerned with four previously mentioned frequency oscillations or bands: ULF, VLF, LF, and HF. For humans ULF, VLF, LF, and $\mathrm{HF}$ frequency bands are defined to be $0-0.0033 \mathrm{~Hz}, 0.003-0.04$ $\mathrm{Hz}, 0.04-0.15 \mathrm{~Hz}$, and $0.15-0.4 \mathrm{~Hz}$ respectively. The ULF and VLF are often ignored due to the lack of long data recordings needed to accurately resolve these frequencies or due to the use of IBI (RR interval) determining techniques that may destroy information within those bands (Clifford, 2012).

LF/HF Ratio - This is the ratio between the power of Low Frequency and High Frequency bands. This measure indicates overall balance between sympathetic and parasympathetic systems. Higher values reflect domination of the sympathetic system, while lower ones - domination of the parasympathetic system. This ratio can be used to help quantify the overall balance between the sym- 
pathetic and parasympathetic systems (Ke, Zulman, Wu, Huang, Thiagarajan, 2016).

Practical computations of the DFT use the FFT for speed advantages. The periodogram, extension of the DFT, is a basic method of estimating power spectral density of a time series. Reducing spectral leakage of the periodogram can be accomplished by incorporating a weighted windowing function w(n), e.g. Hamming and Hanning, to the input series. Finally, in an effort to reduce the variance of the periodogram estimation, the Welch method separates the data series into $\mathrm{N}$ overlapping segments. As with the modified periodogram the Welch method applies a weighting window to reduce spectral leakage, but weighting is applied to each segment. Finally, an averaged PSD is calculated using all segments. Classical power spectrum estimates developed by Bartlett (1948), Blackman and Tukey (1958), and Welch (1967) are examples of methods based on FFT.

Variability (HRV). At the beginning of sleep, LF to HF ratio in HRV signals decreases. This fact is considered as detection of drowsiness (Task Force of the European Society of Cardiology and the North American Society of Pacing and Electrophysiology, 1996). The drowsiness detection (LF/HF ratio) results are affected by several factors: age, gender, origin (Foong, Kai, Chai, Cuntai, Phyo Wai, 2015).

\section{Methods}

\subsection{Measurement goals}

Target of the test stage is to get baseline data from the wearable HR measurement capable devices that were selected initially. The baseline data are the R-R intervals of heartbeats during 5-minute measurement. To ensure that baseline data will be relevant it was decided to do control measurements using certified medical grade ECG registration device Welch Allyn Cardio Perfect Pro and corresponding software (WA CP Workstation) to analyze and interpret ECG data, including $\mathrm{HRV}$ and calculate $\mathrm{LF} / \mathrm{HF}$ ratio. HRVAS software suite is used to calculate the $\mathrm{LF} / \mathrm{HF}$ ratio values from wearable device data. The two software tools require additional calibration to ensure that $\mathrm{LF} / \mathrm{HF}$ ratio values are comparable and results correlate. This can be done by using initial test data The LF/HF output data that is comparable should be obtained by using the same set of mathematical analysis methods (which are partly hidden in the WACP software). The result of drowsiness estimate by LF/HF ratio evaluation should be validated against one of the proven methods. Available methods are EEG measurements and reaction time measurements. EEG measurements require clinical device and isolated environmental conditions - the tests can be held separately without any electronical device in proximity (Corfitsen, 1994). In this case human reaction time measurements can be used to measure reaction time average for 5 quick tests. The reaction time is one of the main risk factors that affect driver's performance (Barrett, 2005). Additional information about the LF/HF ratio calculation can be obtained from existing mobile software that has been approved for HRV feature calculation, so that the HRV features are calculated on the smartphone, and LF/HF ratio values and R-R input data can be stored for later comparison.

\subsection{Data acquisition setup and process}

All day measurement flow steps (see Table I for parameters):

1. Prepare volunteer(s) and setup measurement equipment.

2. Simultaneous measurements of HR (R-R intervals) and ECG from wearable devices for 5 minutes. Volunteer should be in resting position and not moving.

3. Perform reaction tests ( 5 times) after the HR measurements are done.

4. Store measurement results and reaction time average value.
Table 1: Test procedure parameters

\begin{tabular}{|l|l|}
\hline Parameter & Value \\
\hline Test procedure duration & $1-8$ hours \\
\hline Measurement duration & 5 minutes \\
\hline Measurement count & $3-5$ tests \\
\hline $\begin{array}{l}\text { Control method for data valida- } \\
\text { tion }\end{array}$ & ECG 5 minutes measurements \\
\hline $\begin{array}{l}\text { Control method for LF/HF } \\
\text { method validation }\end{array}$ & $\begin{array}{l}\text { Human reaction time measurements } \\
\text { (Average out of 5 tests) }\end{array}$ \\
\hline Number of volunteers & 18 \\
\hline
\end{tabular}

Measurement times are scheduled to cover 1 work day interval with 5 measurement data points and 2-3 hour intervals.

Measurement tool requirements:

1. System must be able to record at least $300 \mathrm{sec}$. (5.min) ECG to analyze it and give at least following parameters:

a) At least R-R interval file (includes each heartbeat interval, not averaged)

b) Analysis, at least dominant High and Low frequency peaks, $\mathrm{H} / \mathrm{L}$ ratio

2. Export data file, as *.txt, *.csv, *.xls or other compatible and easy recoverable format.

3. System must have certification at least according EU Directive 93/42 EEC Medical Devices, and have corresponding CE marking.

4. Positive scientific users' replays required.

The wearable chest belt Polar's H7 is ECG (Electrocardiogram) based heart rate monitor that broadcasts data over Bluetooth (BLE) and is supported by iPhone and Android. For iPhone - Heart Rate Variability Logger requires a Bluetooth Low Energy (also called Bluetooth Smart or BLE or 4.0) heart rate monitor, and the software is capable of logging Heart Rate and also HR features like $\mathrm{LF} / \mathrm{HF}$ ratio and other.

The Welch Allyn Cardio Perfect PRO Cardiograph is specifically intended for the recording of standard 12-lead electrocardiograms of patients. Device connects to a computer using USB connection (Table II).

Table 2: Measurement devices

\begin{tabular}{|c|c|c|}
\hline $\begin{array}{l}\text { Measurement } \\
\text { source }\end{array}$ & $\begin{array}{c}\text { Data acquisition } \\
\text { software }\end{array}$ & $\begin{array}{l}\text { Analysis soft- } \\
\text { ware }\end{array}$ \\
\hline $\begin{array}{c}\text { Polar } \mathrm{H} 7 \text { chest } \\
\text { band }\end{array}$ & $\begin{array}{c}\text { HR \& HRV Logger } \\
\text { for Polar H7 (Android) } \\
\text { Heart Rate Variability } \\
\text { Logger (iOS) }\end{array}$ & $\begin{array}{c}\text { HRVAS v1.0.1 } \\
\text { Heart Rate Varia- } \\
\text { bility Logger } \\
\text { (iOS) } \\
\end{array}$ \\
\hline $\begin{array}{c}\text { ECG - CP Pro } \\
\text { (CardioPerfect Pro) } \\
\text { sampling frequency } \\
600 \mathrm{~Hz}\end{array}$ & $\begin{array}{c}\text { WACP HRV Module } \\
\text { Version 1.6.5.1140 } \\
\text { (Windows) }\end{array}$ & $\begin{array}{c}\text { WACP HRV } \\
\text { Module Version } \\
\text { 1.6.5.1140 (Win- } \\
\text { dows) }\end{array}$ \\
\hline
\end{tabular}

HRVAS software toolbox created in MATLAB is used to analyze $\mathrm{R}-\mathrm{R}$ interval input data. The software is capable of HRV time domain and frequency domain analysis. The output of analysis is $\mathrm{LF} / \mathrm{HF}$ ratio by using Welch, Burg and Lomb-Scargle periodogram. The software is used to interpret R-R data directly since WACP tools used for ECG analysis don't have the feature.

\subsection{Measurement volunteers}

Overall it is necessary to provide data from various volunteer groups. The volunteer set should consist of following:

1. Two gender groups: male and female.

2. Three age groups: 20-24 years, 25-39 years, 30+ years.

Measurement data during test process were obtained from 18 volunteers mostly men in good health condition. The volunteers are from different age and gender groups (Table III). The baseline results for device tests are not considered to be analyzed by the age group distribution as the purpose of tests is to compare statistical data from multiple sensors. The group distribution serves for later analysis of the proposed algorithm in testing stage. Some 
volunteers performed multiple measurement sessions. Results were filtered by the ECG acceptability criteria. There should be at least 3-5 measurements for the volunteer from a single session (one day) that have ECG data and also the ECG data are validated (rejection rate $<1 \%$ of the R-R intervals is accepted). Total of 103 measurements were registered. Below are the accepted measurements after data validation (Figure I).

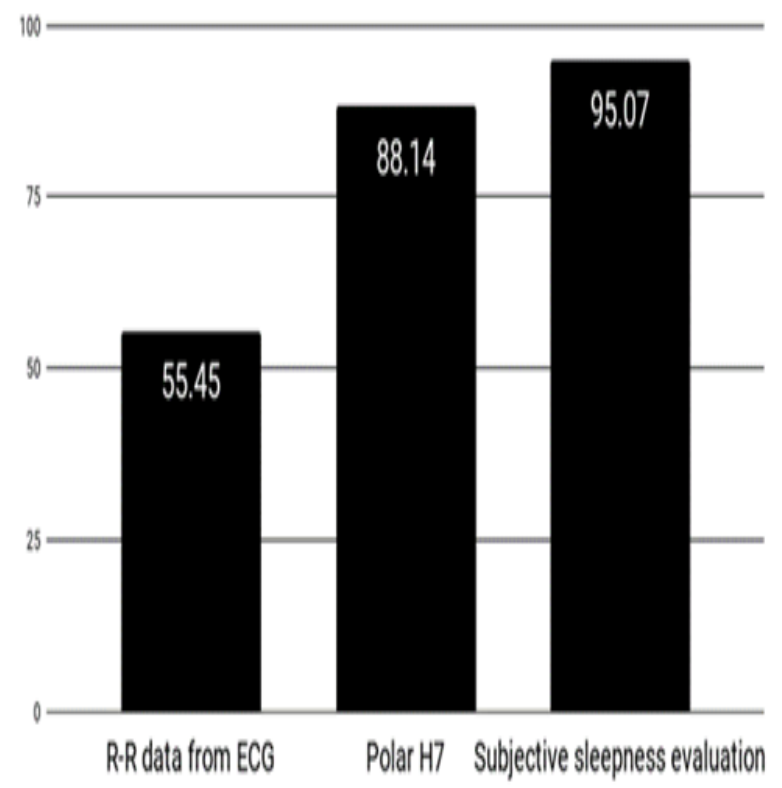

Fig. 1: Percentage of acceptable data from measurements

Table 3: Volunteer age distribution

\begin{tabular}{|c|c|c|c|}
\hline Age group (years) & Male & Female & Total \\
\hline $20-24$ & 8 & 3 & 11 \\
\hline $25-29$ & 5 & 0 & 4 \\
\hline $30-34$ & 1 & 0 & 1 \\
\hline $35-39$ & 1 & 0 & 1 \\
\hline Total & $\mathbf{1 5}$ & $\mathbf{3}$ & $\mathbf{1 8}$ \\
\hline
\end{tabular}

The effect of the result validation is the amount of useable data from ECG results where $44 \%$ of the initial data were unusable. The ECG data error mitigation by using a more controlled measurement approach allowed to reduce the rejection rate for further measurement data (Figure II).

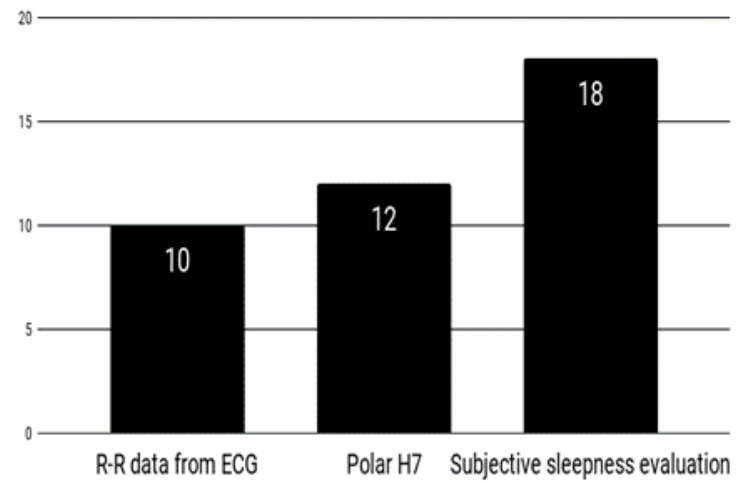

Fig. 2: Number of volunteers with at least 3 valid measurements from a single session

\section{Results}

\subsection{Validation of the $L F / H F$ ratio values from Matlab HRVAS compared with medical ECG and mobile FFT software}

Data (LF/HF ratio values) from Welch Allyn medical ECG device has been compared to values calculated by Matlab HRVAS tool. It is necessary to ensure that the medical equipment software (WA) and Matlab HRVAS tools have comparable results for LF/HF ratio since HRVAS tool is used to process R-R data but WACP is used for discrete ECG processing. The results show complete correlation between Welch Allen DFT method output and HRVAS Burg DFT output. The example values were calculated form 5 measurement data of Volunteer 14 (Figure III) where each data point corresponds to 5 minute sample window spectral analysis of R-R data.

Further, IPhone Heart Rate Variability logger mobile software is used to obtain and process R-R interval data from Polar $\mathrm{H} 7$ wearable ECG based sensor. Both sensors obtain the measurements simultaneously within the given sample intervals. The comparison for LF/HF ratio shows that overall mobile software results do not correlate with Burg DFT processed R-R interval data. This leads to show the values are lower and may use different window function with normalization parameters (Welch or Lomb-Scargale) for frequency analysis of R-R intervals (Figure IV). The difference between results in Figure IV increases data points where the LF to $\mathrm{HF}$ ratio increases and indicates narrowed range for $\mathrm{LF} / \mathrm{HF}$ ratio analysis with the observed mobile software.

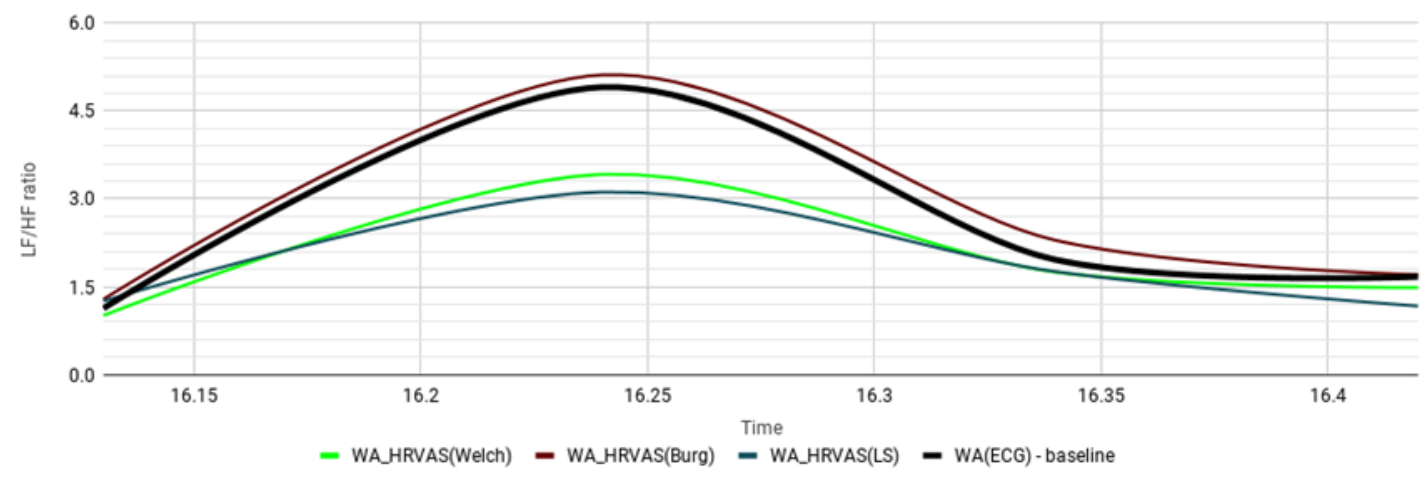

Fig. 3: Measurement comparison Welch Allyn ECG analysis tool with DFT and Matlab HRVAS with three different frequency domain transformation methods 


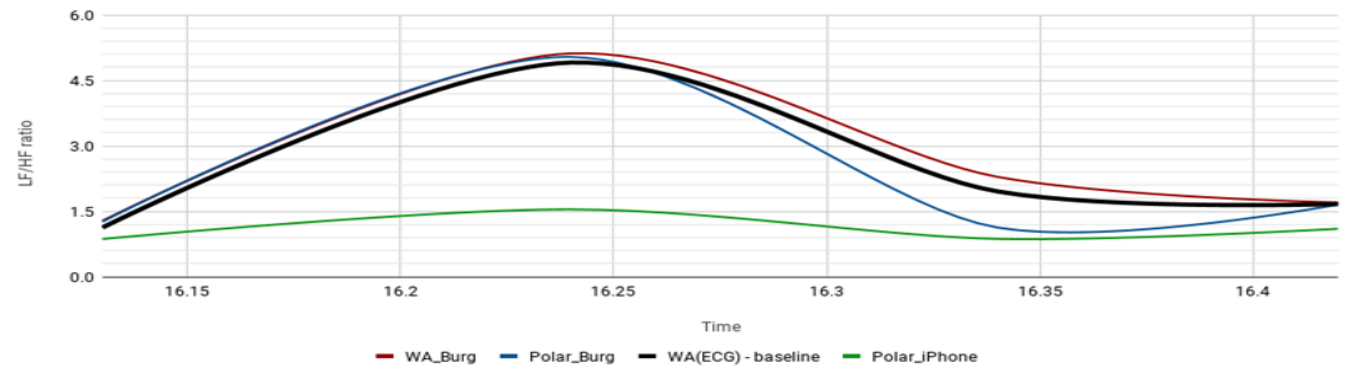

Fig 4: LF/HF ratio comparison mobile software, HRVAS_Burg and WA data from ECG equipment and wearable sensor

\subsection{Statistical validation of the test device - ECG wrist band}

The average results were collected from 10 volunteer tests with 3 measurements for each volunteer (Table IV) with Polar H7 and ECG equipment data exported with Welch Allyn Cardio Perfect software. The results for average R-R intervals differ for only $0.6 \%$ that shows close correlation and indicates that Polar can be used to work with R-R data with ECG equipment. Average dispersion and average help indicating measurements with large difference i.e. Volunteer 2 data have greatest difference. Considering the time synchronization for manual measurements, the results indicate minimal statistical difference.

Polar H7 LF/HF ratio values closely correlate with ECG recordings (Figure V). Results for Volunteer 7 show that Polar H7 can be used with ECG equipment to measure R-R intervals and compare $\mathrm{LF} / \mathrm{HF}$ ratio.

More statistical proof shows summarized average results from 10 volunteers in Table V. Each volunteer data set contains 3 measurements. Each R-R measurement was analyzed with Welch Allyn Cardio Perfect and HRVAS using Burg method for HF/HF ratio. The results in Table $\mathrm{V}$ show that average LF/HF deviation is minimal and confirm high correlation.

Table 4: Comparison of R-R Interval Data from Polar H7 and ECG Equipment

\begin{tabular}{|c|c|c|c|}
\hline & $\begin{array}{c}\text { Average } \\
\text { mean RR }\end{array}$ & $\begin{array}{c}\text { Average } \\
\text { dispersion }\end{array}$ & $\begin{array}{c}\text { Average } \\
\text { standard } \\
\text { deviation }\end{array}$ \\
\hline Volunteer 1 & $0.063 \%$ & $1,320 \%$ & $0.663 \%$ \\
\hline Volunteer 2 & $3,989 \%$ & $28,532 \%$ & $15,733 \%$ \\
\hline Volunteer 5 & $0.249 \%$ & $5,526 \%$ & $2,818 \%$ \\
\hline Volunteer 7 & $0.363 \%$ & $5,081 \%$ & $2,618 \%$ \\
\hline Volunteer 9 & $0.393 \%$ & $14,147 \%$ & $7,569 \%$ \\
\hline Volunteer 10 & $0.115 \%$ & $0.404 \%$ & $0.202 \%$ \\
\hline Volunteer 11 & $0.600 \%$ & $17,190 \%$ & $9,503 \%$ \\
\hline Volunteer 14 & $0.159 \%$ & $3,246 \%$ & $1,662 \%$ \\
\hline Volunteer 15 & $0.343 \%$ & $2,981 \%$ & $1,502 \%$ \\
\hline Volunteer 16 & $0.385 \%$ & $5,065 \%$ & $2,604 \%$ \\
\hline Total average & $\mathbf{0 . 6 6 6 \%}$ & $\mathbf{8 , 3 4 9 \%}$ & $\mathbf{4 , 4 8 7 \%}$ \\
\hline
\end{tabular}

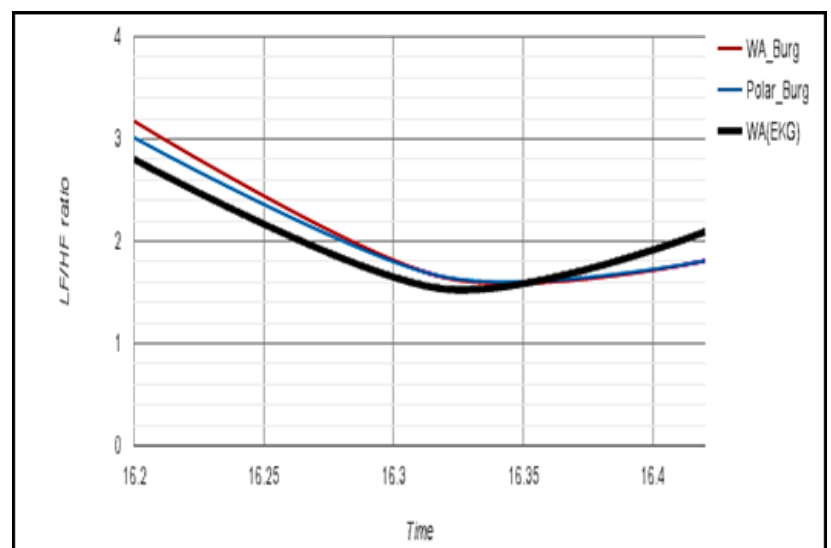

Fig. 5: Polar 7 and ECG calculated LF/HF ratio for Volunteer 7 data with three measurements
Table 5: Statistical data for LF/HF Ratio Based on R-R Data from Polar H7 and Medical ECG Equipment

\begin{tabular}{|c|c|c|c|}
\hline & $\begin{array}{c}\text { Average } \\
\text { dispersion }\end{array}$ & $\begin{array}{c}\text { Average } \\
\text { standard } \\
\text { deviation }\end{array}$ & $\begin{array}{c}\text { Average coef- } \\
\text { ficient of vari- } \\
\text { ation }\end{array}$ \\
\hline Volunteer 1 & 0.75 & 0.77 & 0.09 \\
\hline Volunteer 2 & 0.04 & 0.19 & 0.10 \\
\hline Volunteer 5 & 0.05 & 0.21 & 0.14 \\
\hline Volunteer 7 & 0.02 & 0.14 & 0.07 \\
\hline Volunteer 9 & 0.33 & 0.42 & 0.24 \\
\hline Volunteer 10 & 0.00 & 0.02 & 0.06 \\
\hline Volunteer 11 & 0.01 & 0.06 & 0.05 \\
\hline Volunteer 14 & 0.13 & 0.26 & 0.14 \\
\hline Volunteer 15 & 0.08 & 0.24 & 0.19 \\
\hline Volunteer 16 & 0.00 & 0.06 & 0.08 \\
\hline Total average & $\mathbf{0 . 1 4}$ & $\mathbf{0 . 2 4}$ & $\mathbf{0 . 1 2}$ \\
\hline
\end{tabular}

\section{Conclusion}

There is a statistically confirmed correlation between medical ECG equipment software output and Matlab HRVAS output when the LF/HF ratio is calculated based on Burg FFT spectral analysis of R-R interval data. The Matlab HRVAS software can be further used for ECG based R-R interval data frequency analysis from other sensors or data sources.

Medical ECG equipment measurements indicated $55.45 \%$ of statistical data set have less than $3 \%$ invalid R-R interval samples used for frequency analysis. The reviewed approach of using Matlab HRVAS software for R-R interval analysis of public medical ECG database enables research with extended statistical data set and analysis of subjects with common properties or expertannotated cardiological diagnoses.

To get Polar H7 R-R interval data and then calculate LF/HF ratio, the IPhone Heart Rate Variability logger software application was used. The results of the mobile software comparison show that other software applied normalization of the frequency narrows the differentiation when LF to LF ratio increases influencing the estimation of disbalance between the two ECG features. However, it is possible to adjust the parameters of Matlab HRVAS software to obtain results comparable to discrete and proprietary ECG spectral analysis algorithms.

The results of LF/HF ratio values from wearable ECG sensor closely correlate with data extracted from medical ECG device recordings obtained from Welch Allyn Cardio Perfect software that enables the use of single electrode wearable device data validation with medical control devices.

\section{References}

[1] Aubert, A. E., Seps, B., \& Beckers, F. (2003). "Heart rate variability in athletes". Sports Medicine, pp. 889-919.

[2] Barrett, P. R. (2005). "Interactions between moderate alcohol consumption and sleepiness: the effect on driver performance". Retrieved from https://dspace.lboro.ac.uk/dspacejspui/bitstream/2134/15748/1/Thesis-2005-Barrett.pdf

[3] Clifford, G. D. (2012). Signal Processing Methods for Heart Rate Variability Analysis. 27. St. Cross College. Retrieved from 
http://web.mit.edu/ gari/www/papers/GDCliffordThesisAbstract.pd $\mathrm{f}$

[4] Corfitsen, M. T. (1994, Oct 26). Tiredness and visual reaction time among young male nighttime drivers: a roadside survey. Accident Analysis \& Prevention, pp. 617-624

[5] Fenton-O'Creevy, M., \& et al. (2012). "Emotion regulation and trader expertise: Heart rate variability on the trading floor.", Journal of Neuroscience, Psychology, and Economics, p. 227.

[6] Foong, R., Kai, A., Chai, Q., Cuntai, G., \& Phyo Wai, A. (2015) An Analysis on Driver Drowsiness Based on Reaction Time and EEG Band Power. Conference proceedings: Annual International Conference of the IEEE Engineering in Medicine and Biology Society. IEEE Engineering in Medicine and Biology Society. Retrieved from https://www.ncbi.nlm.nih.gov/pubmed/26738144

[7] Ke, K.-W., Zulman, M. R., Wu, H.-T., Huang, Y.-F., \& Thiagarajan, J. (2016). Drowsiness Detection System Using Heartbeat Rate in Android-Based Handheld Devices, First International Conference on Multimedia and Image Processing (ICMIP)., (p. 99).

[8] Kusserow, M., Amft, O., \& Tröster, G. (2008). "Analysis of heart stress response for a public talk assistant system". Ambient Intelligence, pp. 326-342.

[9] Malik, M., Hnatkova, K., \& Camm, A. J. (1995). "Practicality of postinfarction risk assessment based on time-domain measurement of heart rate variability". Armonk, NY: Futura Pub. Co.

[10] Medicore. (2005). "Heart rate Variability Analysis System". (3.0) Medicore. Retrieved from http://medicore.com/download/HRV_clinical_manual_ver3.0.pdf

[11] Nolan, J., \& et al. (1998). "Prospective study of heart rate variability and mortality in chronic heart failure results of the United Kingdom heart failure evaluation and assessment of risk trial (UKHeart)". Circulation, pp. 1510-1516.

[12] Task Force of the European Society of Cardiology and the North American Society of Pacing and Electrophysiology. (1996). "Heart rate variability standards of measurement, physiological interpretation, and clinical use". Circulation, pp. 1043-1065. Retrieved from https://doi.org/10.1161/01.CIR.93.5.1043

[13] Van Doornen, L. J., de Geus, E. J., \& Vrijkotte, T. G. (2000). "Effects of work stress on ambulatory blood pressure, heart rate, and heart rate variability". Hypertension, pp. 880-886. 